\title{
A short note on fuzzy relational inference systems ${ }^{\mathrm{I}}$
}

\author{
Martin Štěpnička ${ }^{\mathrm{a}, *}$, Balasubramaniam Jayaram ${ }^{\mathrm{b}}$, Yong $\mathrm{Su}^{\mathrm{c}}$ \\ ${ }^{a}$ Institute for Research and Applications of Fuzzy Modeling, University of Ostrava, \\ CE IT4Innovations, 30. dubna 22, 70103 Ostrava, Czech Republic \\ ${ }^{b}$ Department of Mathematics, Indian Institute of Technology Hyderabad, Kandi 502 285, \\ Telangana, India \\ ${ }^{c}$ School of Mathematics, Shandong University, Shandong Sheng, Jinan, China
}

\begin{abstract}
This paper is a short note contribution to the topic of fuzzy relational inference (FRI) systems and the preservation of their desirable properties. It addresses the two main fuzzy relational inferences - compositional rule of inference (CRI) introduced by Zadeh and the Bandler-Kohout subproduct (BK-Subproduct) - and their combination with two fundamental fuzzy relational models of fuzzy rule bases, namely, the Mamdani-Asilian and the implicative models.

The goal of this short note article, is threefold. Firstly, we show that one of the results provided in [37], namely the robustness related to the combination of BK-subproduct and implicative fuzzy rule base model, was not proven correctly as it used an incorrect lemma. However, we will show that the result itself is still valid as an alternative and valid proof will be provided. Secondly, we present some of the results provided already in [23] and later on in other works in the perspective of the preservation of the desirable properties. Finally, we provide readers with a brief yet important discussion on consequences of these results in the light of the theoretical as well as practical aspects of building fuzzy inference systems.
\end{abstract}

\footnotetext{
IThe first author declares partial support by the NPU II project LQ1602 "IT4Innovations excellence in science" provided by the MŠMT. The second author would like to acknowledge the project CSIR HRDG-INDIA (25 (0235)/14/EMR II) for financial support.

${ }^{*}$ Corresponding author. Tel.: +420 59709 1403; fax: +420 596120478.

Email addresses: Martin.Stepnicka@osu.cz (Martin Štěpnička), jbala@iith.ac.in (Balasubramaniam Jayaram), yongsu88@163.com (Yong Su)
} 
Keywords: Fuzzy relational inference systems, Compositional rule of inference, Bandler-Kohout subproduct, Fuzzy relational equations, Robustness of inference.

\section{Introduction}

The notion of fuzzy inference systems (or fuzzy rule based systems) denotes systems incorporating the fundamental idea of ZADEH to imitate the human way of thinking based on fuzzy rules, see e.g. [41]. The application potential has been proven to be huge and the list of successful applications as well as deep theoretical studies would quickly exceed the page limit and hence let us only mention the very first by MAMDANI AND ASSILIAN [21] as a tribute to the authors.

If we restrict our focus on the fuzzy relational inference ${ }^{1}$, we face the following situation. Let $X, Y$ be non-empty classical sets (universes) and the sets of all fuzzy sets on $X(Y)$ are denoted by $\mathcal{F}(X)$ (resp. $\mathcal{F}(Y)$ ). A fuzzy (IF-THEN) rule base is a set of $n$ fuzzy rules usually given in the following form:

$$
\text { IF } x \text { is } A_{i} \quad \text { THEN } y \text { is } B_{i}, \quad i=1, \ldots, n,
$$

where the fuzzy sets $A_{i} \in \mathcal{F}(X)$ and $B_{i} \in \mathcal{F}(Y)$, represent some properties.

Given an observation $A^{\prime} \in \mathcal{F}(X)$, a corresponding output fuzzy set $B^{\prime} \in$ $\mathcal{F}(Y)$, is inferred with the help of fuzzy inference mechanism and the given fuzzy rule base. Thus, an inference mechanism may be generally viewed as an arbitrary mapping from $\mathcal{F}(X)$ to $\mathcal{F}(Y)$ [11, 12]. However, as mentioned above, we will focus on the fuzzy relational inference mechanism initiated by ZADEH in 1973.

Fuzzy relational inference mechanisms use a fuzzy relation $R$ to model a given fuzzy rule base. Here, a fuzzy IF-THEN rule of the form (1) is represented as a fuzzy relation $R \in \mathcal{F}(X \times Y)$. Then, given a fact $x$ is $A^{\prime}$, the inferred output $y$ is $B^{\prime}$ is obtained as a composition of $A^{\prime}$ and $R$, i.e.,

$$
B^{\prime}=A^{\prime} @ R,
$$

\footnotetext{
${ }^{1}$ Let this restriction be not viewed as a lack of respect to other approaches but rather as a necessary technical narrowing of the class of problems we are facing. The authors are fully aware of other approaches, mainly e.g. Similarity Based Reasoning [32]. It is also worth mentioning that under certain conditions, an equivalent fuzzy relation based description of some of these inference systems can be built, see [4] so, the outcomes of this article are still also highly related to these other systems.
} 
where $A^{\prime} \in \mathcal{F}(X), B^{\prime} \in \mathcal{F}(Y)$ and @ is a fuzzy relational composition or more precisely an image of the fuzzy set $A^{\prime}$ under the fuzzy relation $R$ that is derived from the fuzzy relational composition.

Clearly, for different models $R$ of the fuzzy rules and for different compositions @ we obtain different fuzzy relational inference systems, often with varying properties. Let us recall two most commonly employed classes of fuzzy relations $R$ to model a given fuzzy rule base and two well-established fuzzy relational inference mechanisms based on different fuzzy compositions.

Most often used class of models stems from the already mentioned successful application by MAMDANI AND AssiLian [21] and uses the following fuzzy relation $\check{R} \in \mathcal{F}(X \times Y)$ :

$$
\check{R}(x, y)=\bigvee_{i=1}^{n}\left(A_{i}(x) * B_{i}(y)\right), \quad x \in X, y \in Y .
$$

This, say Cartesian product approach, originally used the minimum operation $(*=\wedge)$, and it has been later on generalized for other conjunctors. It makes sense to consider this class of models restricted to arbitrary yet meaningful and rich enough class of operations, e.g. left-continuous t-norms $[17]$.

Alternatively, in order to capture the conditional form of fuzzy rules (1), the following fuzzy relation $\hat{R} \in \mathcal{F}(X \times Y)$ :

$$
\hat{R}(x, y)=\bigwedge_{i=1}^{n}\left(A_{i}(x) \rightarrow B_{i}(y)\right), \quad x \in X, y \in Y
$$

can also be chosen to model the fuzzy rule base. It deals with a correctly defined fuzzy implication $\rightarrow[1]$, which is often a residual implication derived from a left-continuous t-norm $*$.

With respect to the short-note nature of this article, we do not find it appropriate to stress the difference between both models in detail but as this issue deserves importance, we provide readers with relevant references $[3,8,26]$ and we add an important note, that both the models have sound logical foundations, see e.g. [9, 13, 27].

Two of the commonly employed fuzzy relational compositions @ are the sup $-*$ and inf $-I$ compositions [18], which when employed lead to the following fuzzy relational inferences. The Compositional Rule of Inference (CRI) 
introduced by ZADEH [41], infers the output $B^{\prime}$ in the following way:

$$
B^{\prime}(y)=\bigvee_{x \in X}\left(A^{\prime}(x) * R(x, y)\right), \quad y \in Y
$$

where $*$ is a again a conjunctor, typically a (left-continuous) t-norm [17]. For CRI scheme, we use the following notation:

$$
B^{\prime}=A^{\prime} \circ R
$$

The second inference, based on the Bandler-Kohout subproduct (BKSubproduct, for short) [2], was up to our best knowledge firstly used by PEDRYCZ [28] and although it is much less frequently used, it has started to attract attention in the recent years $[20,22,34,38]$. The output $B^{\prime}$ inferred by the BK-Subproduct is obtained as follows:

$$
B^{\prime}(y)=\bigwedge_{x \in X}\left(A^{\prime}(x) \rightarrow R(x, y)\right), \quad y \in Y
$$

where $\rightarrow$ is a residual implication. For BK-Subproduct, we use the following notation to indicate the scheme:

$$
B^{\prime}=A^{\prime} \triangleleft R
$$

As one can see, for the sake of simplicity and clarity, everything may be elaborated in the framework of residuated lattice (see, e.g., [27]) $\mathcal{L}=$ $(L, \wedge, \vee, *, \rightarrow, 0,1)$ as the underlying algebraic structure. We assume the readers to be familiar with basic properties of these structures and also with the fact, that each left-continuous t-norm $*$ generates such a structure [5] so, we only add references to relevant sources $[13,27]$. The basic properties are also listed in [37], of which the following are essential in the sequel:

$$
\begin{aligned}
\bigvee_{i \in \mathcal{I}} a_{i} \rightarrow b & =\bigwedge_{i \in \mathcal{I}}\left(a_{i} \rightarrow b\right), \\
a \rightarrow(b \rightarrow c) & =(a * b) \rightarrow c=(b * a) \rightarrow c, \\
(a \rightarrow b) * c & \leq a \rightarrow(b * c) .
\end{aligned}
$$

For better understanding, let us adopt the notation from [29] and later on used also in [37] and consider the following structure

$$
S=\left(X, Y,\left\{A_{i}, B_{i}\right\}_{i=1, \ldots, n}, \mathcal{L}, @\right),
$$


where $X, Y$ are the universes, $A_{i} \in \mathcal{F}(X), B_{i} \in \mathcal{F}(Y)$ for all $i=1, \ldots, n$ are the antecedent and consequent fuzzy sets in the fuzzy rule base, @ : $\mathcal{F}(X) \times$ $\mathcal{F}(X \times Y) \rightarrow \mathcal{F}(Y)$ is the chosen inference (fuzzy relational composition) and finally, $\mathcal{L}$ is the fixed residuated lattice on the unit interval $[0,1]$ that provides us with the involved operations.

By the choice of the fuzzy relation $R$ modeling the fuzzy rule base and by the choice of $@$, we define a fuzzy function $f_{R}^{@}(A): \mathcal{F}(X) \rightarrow \mathcal{F}(Y)$ such that $f_{R}^{@}(A)=A @ R$, for an arbitrary $A \in \mathcal{F}(X)$.

\section{Motivation}

The goals of this article, that richly stems from findings in [37, 23] and in [39], is threefold. So, let us firstly recall what was provided in the above cited works.

In [37], the authors demonstrated, that BK-Subproduct is at least as good as well-known and much more often used CRI. The authors recalled, that the crucial property, that is interpolativity (i.e. preservation of modus ponens) can be ensured under the very same conditions for both inferences and that in the case of the BK-Subproduct, the interpolativity is also equivalent to the "continuity" as it has been defined and proved in [29]. Further, they have shown, that the other advantageous properties, e.g. robustness, equivalence of FITA and FATI inference strategies or an existence of hierarchical inference algorithm that significantly reduces the computational costs, are not dependent on the chosen inference but rather on an appropriate combination of the inference and the fuzzy rule base model. In particular, for the three latter properties, one has to either combine CRI with the Mamdani-Assilian model $\check{R}$ or BK-Subproduct with the implicative model $\hat{R}$. Let us note that these facts were already known for the first combination [16, 14] and the authors of [37] proved them for the latter one in order to show that both approaches are equally good.

Now, the threefold goal of this paper is as follows.

- Firstly, we show, that one of the results provided in [37], namely the robustness related to the combination of BK-subproduct and implicative fuzzy rule base model, was not proved correctly as it used an incorrect lemma. However, the result itself is still valid and an alternative valid proof is provided. 
- Secondly, we present some of the results provided already by Moser AND NAVARA [23] and later on by ŠTĚPničKA AND MANDAL [39] in the perspective of the preservation of the desirable properties discussed in our previous paper.

- Finally, we provide readers with a brief yet important discussion on consequences of these results in the light of the theoretical as well as practical aspects of building fuzzy inference systems.

The structure of the rest of the paper clearly follows these three goals.

\section{Goal 1: Robustness of BK-Subproduct and implicative models}

In [16], KlaWonn and Castro constructed their approach to the notion of robustness of fuzzy inference systems in a natural way. In particular, they shown that (cit. [16]) "the output obtained from CRI for a given input fuzzy set $A^{\prime}$ does not change if we substitute $A^{\prime}$ by its extensional hull $\widehat{A}^{\prime \prime \prime}$. The important assumption is, that the input $A^{\prime}$ is extensional w.r.t the same fuzzy equivalence relation ${ }^{2}$ to which also the antecedent fuzzy sets are extensional. Indeed, "a fuzzified input does not change the outcome of a rule as long as the fuzzy set obtained by the fuzzification is contained in the extensional hull of the original crisp input value" [16]. Furthermore, the authors finally conclude that it does not make sense to measure more exactly than the indistinguishability admits. This approach formalizes the robustness of the inference in scenarios where there can be slight discrepancies between the intended input and the actual input.

The result was originally provided for CRI with both $\check{R}$ and $\hat{R}$, but only for a single rule [16]. In [37] it was generalized for more rules, which, on the other hand, required the restriction to the use of $\check{R}$ only. It was formulated as follows.

Theorem 1. ([37], Theorem 3.20) Let $S=\left(X, Y,\left\{A_{i}, B_{i}\right\}_{i=1, \ldots, n}, \mathcal{L}, \circ\right)$ be a structure for fuzzy rules (1). Let $E$ be a fuzzy equivalence relation on $X$ with respect to which each $A_{i}$ is extensional for $i=1, \ldots, n$. If $A^{\prime} \in \mathcal{F}(X)$,

\footnotetext{
${ }^{2}$ With respect to the limited size of a short note paper, we again assume that the readers are familiar with the basic definitions of fuzzy equivalence relations (also similarity or indistinguishability), and the extensional hull.
} 
then $A^{\prime} \circ \check{R}=\widehat{A}^{\prime} \circ \check{R}$, where $\widehat{A}^{\prime}$ is the extensional hull of $A^{\prime}$ w.r.t. the fuzzy equivalence relation $E$.

Furthermore, in [37] the authors provided an analogous result for the combination of BK-Subproduct and the implicative model of a fuzzy rule base $\hat{R}$, which we recall below.

Theorem 2. ([37], Theorem 3.23) Let $S=\left(X, Y,\left\{A_{i}, B_{i}\right\}_{i=1, \ldots, n}, \mathcal{L}, \triangleleft\right)$ be a structure for fuzzy rules (1). Let $E$ be a fuzzy equivalence relation on $X$ with respect to which each $A_{i}$ is extensional, for $i=1, \ldots, n$. If $A^{\prime} \in \mathcal{F}(X)$, then $A^{\prime} \triangleleft \hat{R}=\widehat{A}^{\prime} \triangleleft \hat{R}$.

These generalizations to multiple rules provided by Theorems 1 and 2 are on the other hand restrictive w.r.t. the chosen combination of the inference mechanism and the fuzzy rule base model. As we have mentioned above, KLAWONN and CASTRO in [16] proved that, assuming the CRI inference, for a single rule the robustness is preserved no matter the choice of the model. Although a single rule fuzzy rule base is useless in practice, there is an undoubted importance of the result for so-called FITA inference strategies, where instead of a single fuzzy relation representing the whole fuzzy rule base (1), one takes a single fuzzy relation for every single fuzzy rule and performs $n$ inferences, each inference only with help of a single fuzzy rule. In other words, in FITA strategies, the fuzzy rule base (1) is viewed as $n$ fuzzy rule bases of a single rule and KLAWONN and CASTRO actually provide a strong result stating, that in this case, one may deal with both the conjunctive as well as implicative model of a fuzzy rules base.

This significance of this result can hardly be exaggerated, bearing in mind that many applications adopt the FITA strategies rather than FATI strategies (first-aggregate-then-infer) [10] encoded usually in the fuzzy relational representation by $\check{R}$ or $\hat{R}$. Therefore, authors in [37] provided again an analogous result for the BK-Subproduct, which we recall below.

Theorem 3. ([37], Theorem 3.22) Let $S=(X, Y,\{A, B\}, \mathcal{L}, \triangleleft)$ be a structure for a single fuzzy rule. Let $E$ be a fuzzy equivalence relation on $X$ with respect to which $A$ is extensional. Let $A^{\prime} \in \mathcal{F}(X)$ be any fuzzy set, then $A^{\prime} \triangleleft \hat{R}=\widehat{A}^{\prime} \triangleleft \hat{R}$ and $A^{\prime} \triangleleft \check{R}=\widehat{A^{\prime}} \triangleleft \check{R}$.

The authors built the proof of the second part of the Theorem, in particular of the equality

$$
A^{\prime} \triangleleft \check{R}=\widehat{A}^{\prime} \triangleleft \check{R}
$$


on an alternative representation of an extensional hull of a fuzzy set, that was also provided in [37]. However, this representation was not proved correctly. We repeat the incorrect proposition and we show, why it cannot be correct.

Proposition 4. (Proposition 3.21 in [37], INCORRECT!) Let $\mu \in \mathcal{F}(X)$ and $E$ a fuzzy equivalence relation on $X$. Then the extensional hull of $\mu$ may be expressed as follows

$$
\widehat{\mu}(x)=\bigwedge\{E(x, y) \rightarrow \mu(y) \mid y \in X\} .
$$

Indeed, if $\widehat{\mu}(x)$ was given as above, then it would necessarily lead to the following

$$
\widehat{\mu}(x)=\bigwedge_{y \in X} E(x, y) \rightarrow \mu(y) \leq E(x, x) \rightarrow \mu(x)=\mu(x)
$$

while as we know, the opposite inequality, i.e., $\mu(x) \leq \widehat{\mu}(x)$ holds. This easily demonstrates, why the above recalled proposition cannot hold in general.

However, although the equality formulated in Proposition 4 (Proposition 3.21 in [37]) does not hold, it does not necessarily mean that the second part of Theorem 3 (Theorem 3.22 in [37]) is not valid anymore. In fact, the opposite is true, as we show below.

Proof of Theorem 3. (of Theorem 3.22 in [37])

The first equality

$$
A^{\prime} \triangleleft \hat{R}=\widehat{A^{\prime}} \triangleleft \hat{R}
$$

is proven in [37] and hence we concentrate on proving the second equality

$$
A^{\prime} \triangleleft \check{R}=\widehat{A^{\prime} \triangleleft \check{R}}
$$

As the antitonicity of residuated implication $\rightarrow$ ensures that

$$
A^{\prime} \triangleleft \check{R} \geq \widehat{A^{\prime}} \triangleleft \check{R}
$$

holds, it is sufficient to prove the other inequality, namely

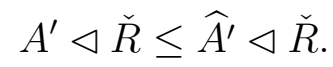

Recall that, for any $y \in Y$,

$$
\left(\widehat{A}^{\prime} \triangleleft \check{R}\right)(y)=\bigwedge_{x \in X}\left(\widehat{A}^{\prime}(x) \rightarrow(A(x) * B(y))\right) .
$$


Taking the right hand side of the equality, then for any $x \in X$, we have

$$
\begin{aligned}
& \widehat{A}^{\prime}(x) \rightarrow(A(x) * B(y)) \\
& =\left(\bigvee_{z \in X}\left(A^{\prime}(z) * E(x, z)\right)\right) \rightarrow(A(x) * B(y)) \\
& =\bigwedge_{z \in X}\left(\left(A^{\prime}(z) * E(x, z)\right) \rightarrow(A(x) * B(y))\right) \\
& =\bigwedge_{z \in X}\left(A^{\prime}(z) \rightarrow(E(x, z) \rightarrow(A(x) * B(y)))\right) \\
& \geq \bigwedge_{z \in X}\left(A^{\prime}(z) \rightarrow((E(x, z) \rightarrow A(x)) * B(y))\right) \\
& \geq \bigwedge_{z \in X}\left(A^{\prime}(z) \rightarrow(A(z) * B(y))\right) \quad\lceil\text { by definition of } E \\
& =\left(A^{\prime} \triangleleft \check{R}\right)(y) .
\end{aligned}
$$

which proves the desired inequality and thus, the whole Theorem.

This proof closes the first goal of the paper related to robustness of fuzzy inference systems with the use of the BK-Subproduct $\triangleleft$ as the inference mechanism. As we have shown, the previous results are still valid, however, the proof has to be constructed in a different way.

\section{Goal 2: Preservation of desirable properties}

This section focuses on the preservation of desirable properties of the above mentioned fuzzy inference systems. Mainly, it stems from the axiomatization provided by Moser AND NAVARA [23] and later on followed in $[39,33]$. In fact, the most important results actually stem partly from even older publications $[15,25]$ related to the solvability of fuzzy relational equations and thus, consequently, to interpolativity alias preservation of modus ponens. Note that this property is perhaps the most crucial one for fuzzy inference systems incorporating fuzzy inputs. Let us only briefly recall the most important results from the field of the solvability of fuzzy relational equations that are gathered from the sources referred above and from the following ones [7, 31, 28, 6].

The main idea behind interpolativity is expressed by the following equality $f_{R}^{@}\left(A_{i}\right)=B_{i}$ which says nothing else but the fact that if an input $A^{\prime}$ is 
equal to one of the antecedents, say to $A_{i}$, the inferred output $B^{\prime}$ has to be equal to the respective consequent $B_{i}$. This leads us to deal with a system of fuzzy relational equations [31] where

$$
A_{i} @ R=B_{i}, \quad i=1, \ldots, n
$$

is solved with respect to an unknown $R \in \mathcal{F}(X \times Y)$.

Theorem 5. System (13) with @ = $=(@=\triangleleft)$ is solvable if and only if $\hat{R}(\check{R})$ is a solution of this system. In the case of solvability, $\hat{R}(\check{R})$ is the greatest (least) solution of (13) with $@=\circ(@=\triangleleft)$.

Theorem 5 actually states that the implicative model $\hat{R}$ (or MamdaniAssilian model $\check{R}$ ) should be the first choice whenever dealing with the inference mechanism modelled by $\circ$ (or $\triangleleft$, respectively). However, if there exist some reasons, for example preservation of robustness, lower computational complexity or the existence of hierarchical inference that is identical to the non-hierarchical one [37, 14], the combination of Mamdani-Assilian model $\check{R}$ and the CRI inference o (implicative model $\hat{R}$ and the BKS inference $\triangleleft$ ) can be used as well without harming the preservation of modus ponens. Indeed, some additional assumptions, specified in the Theorem below, have to be imposed.

Theorem 6. [15, 25] Let all $A_{i}$ for $i=1, \ldots, n$ be normal. Then $\check{R}(\hat{R})$ is a solution of (13) with $@=\circ(@=\triangleleft)$ if and only if the condition

$$
\bigvee_{x \in X}\left(A_{i}(x) * A_{j}(x)\right) \leq \bigwedge_{y \in Y}\left(B_{i}(y) \leftrightarrow B_{j}(y)\right)
$$

holds for any $i, j \in\{1, \ldots, n\}$.

Now, let us focus on the impact of Theorem 6 . On one hand, it gives a positive result. On the other hand, condition (14) contains a hidden trap. This condition may be easily fulfilled for t-norms with zero divisors such as the Łukasiewicz t-norm, while in the case of t-norms without zero divisors, meeting the criterion is not so easy.

Indeed, on the one hand if you consider the Eukasiweicz t-norm and, moreover, you assume the antecedent fuzzy sets to meet the Ruspini partition axioms [30], which is a well-known sufficient condition for the solvability of both systems of fuzzy relational equations [36, 35], left-hand side of (14) 
will equal to zero and thus, the criterion (14) will be trivially fulfilled. Consequently, for this particular algebra, the Ruspini partition will not only be a sufficient condition, but it will be even sufficient for the use of the models, that may provide some computational or robustness advantages.

On the other hand, considering the t-norm without zero divisors, such as the Gödel t-norm (minimum) or the Goguen t-norm (product), one encounters a peculiar situation. It can be easily checked, that the right-hand side of (14) equals to zero whenever the consequents $B_{i}$ and $B_{j}$ have mutually different support. Only in case of totally equal supports of all consequents, the right-hand side is non-trivially positive. However, having totally the same consequent supports is not a desirable condition at all. Now, if we allow the right-hand side to be equal to zero by using consequents with different supports, we need to set up the antecedents in such a way that also the left-hand side of (14) equals to zero. Then again, recall that $*$ is now a t-norm without zero divisors which means that the antecedents would have to be disjoint in a crisp sense! Consequently, either we have to deal with undesirable consequents, or with antecedents that do not overlap. Thus we are forced to work with sparse rule bases, which is not desirable either, however sophisticated the fuzzy interpolation techniques that we employ may be [19, 40].

It was Moser And Navara [23] who pointed out this drawback. They constructed three natural axioms for "well-behaving" fuzzy inference systems. The first one was the often mentioned interpolativity, i.e. model $R$ has to solve the corresponding system of fuzzy relational equations; the second one was the significance of outputs produced by normal inputs; and the third one was a sort of local monotonicity.

Let us concentrate on the first two axioms, as the last one is usually easy to satisfy $[23,24,39,33]$. The first one is clear and should be fulfilled every time. The second one actually says that if the input is normal, the output should be meaningful, providing some valuable information. The extreme case of non-fulfillment of this axiom is the empty fuzzy set equal to 0 on the whole output universe in the case of Mamdani-Assilian systems or, the universal fuzzy set attaining normality on the whole output universe in the case of implicative rules. These extreme cases are exactly the outputs we obtain whenever we deal with a sparse fuzzy rule base and the input does not fire any of the fuzzy rules. This may easily happen in the case of a sparse fuzzy rule base and the normality of the fuzzy input does not help at all. One would have to use one of the above cited fuzzy interpolation techniques $[19,40]$ but then she/he would not work with fuzzy relational interpretation 
encoded in $\hat{R}$ or $\check{R}$ anymore.

As we have mentioned, due to Theorem 6 , for t-norms without zero divisors and for consequents with different supports, one may ensure the interpolativity only in the case of a sparse fuzzy rules base. This fact is mirrored in the result provided by MOSER AND NAVARA in [23] which essentially states that for the CRI inference and the Mamdani-Assilian model $\check{R}$, assuming the t-norm without zero divisors, the first two axioms cannot be satisfied simultaneously.

This unpleasant fact, that in some cases the two axioms may never be satisfied simultaneously, has been shown also for the combination of the BKSubproduct and the implicative fuzzy rule base model $\hat{R}$, see $[39,33]^{3}$.

\section{Goal 3: Discussion on practical settings}

The third goal of this article is to briefly discuss the impact of the findings presented above to appropriate practical settings of fuzzy rule based systems.

Note that in [37] the authors have shown that BK-Subproduct, although much less known and often ignored, as an inference mechanism is as appropriate as the much more frequently used CRI. Although one of the findings in [37] was not proven correctly, we provided readers with an alternative proof showing that the stated property still holds and thus nothing changes on the above statement about the equal practical importance of both - BKSubproduct and CRI.

This result was supported by the secondary message of the article [37] which was that most of the advantages, such as lower computational costs or robustness, do not stem from the choice of the inference mechanism itself but rather from the choice of a favorable combination of the inference mechanism and the fuzzy relational model of a fuzzy rule base.

However, one has to be careful in order to preserve fundamental theoretical properties such as interpolativity, that actually entitle us to use the inference mechanism in the context of logically motivated reasoning. In the

\footnotetext{
${ }^{3}$ Note, that for the use of the implicative model $\hat{R}$, the original second axiom provided in [23] had to be modified in order to capture the same property. Indeed, these two approaches are dual [8] and so, the axioms have to be also dually formulated. Therefore, the universal fuzzy set replaces the empty fuzzy set in the axiom in order to determine a non-significant output.
} 
light of other results $[15,23,24,39,33]$ one has to seriously take into account this warning while building a fuzzy inference system. The choice of basic elements of such systems, such as the underlying algebraic structure of the connectives or fuzzy sets partitioning the input universe $X$, seem to be of even more crucial importance.

In particular, one should consult each fuzzy inference system with basic rules already before the building phase of such a system. For the sake of clarity, we list and discuss them briefly in order to explain their importance.

1. If there is no particular reason for reducing computational costs, for using a hierarchical inference ${ }^{4}$ or for assuring the robustness in the "KLAWONN-CASTRO" sense, it is a safe choice to use the combination of $\hat{R}$ and CRI or, of $\check{R}$ and the BK-Subproduct.

2. If there is a reason to use one of the theoretically less preferable combinations, i.e., either $\hat{R}$ with BK-Subproduct or $\tilde{R}$ with CRI, one should not consider using algebras without zero divisors, viz., Gödel, product, etc., for the interpretation of the used connectives. Lukasiewicz or other t-norms with zero divisors can provide an appropriate setting.

3. If the above choice of the usage of the combination of $\hat{R}$ with BKSubproduct or $\check{R}$ with CRI has been made because of strong reasons, then assuming the well-chosen (e.g. Eukasiewicz) algebra, one should carefully choose a way to build antecedent fuzzy sets that preserve the desirable properties. The choice of the underlying algebra is not sufficient, it only gives a chance. Only after satisfying some other assumptions, e.g. forming an appropriate (e.g. Ruspini) partition, the inequality (14) is satisfied and the interpolativity is preserved without harming the other Moser-Navara axioms. Only then can one fully harness the power of all the advantages described in detail in [37] that rise out of this setting.

${ }^{4}$ Note that either of the following, viz., the equivalence of FITA and FATI strategies or the existence of an equivalent hierarchical inference mechanism ensures lower computational costs. However, the second approach brings yet another advantage - that of easier interpretability as it actually reduces the number of input variables for the price of a greater number of fuzzy rule bases. 


\section{References}

[1] M. Baczyński, B. Jayaram, Fuzzy Implications, Springer-Verlag, Heidelberg, 2008.

[2] W. Bandler, L. Kohout, Semantics of implication operators and fuzzy relational products, International Journal of Man-Machine Studies 12 (1) (1980) $89-116$.

[3] U. Bodenhofer, M. Daňková, M. Štěpnička, V. Novák, A plea for the usefulness of the deductive interpretation of fuzzy rules in engineering applications, in: Proc. 16th IEEE Int. Conf. on Fuzzy Systems, London, 2007.

[4] B. Bouchon-Meunier, R. Mesiar, C. Marsala, M. Rifqi, Compositional rule of inference as an analogical scheme, Fuzzy Sets and Systems 138 (1) (2003) $53-65$.

[5] R. Bělohlávek, Fuzzy relational systems: Foundations and principles, Kluwer Academic, Plenum Press, Dordrecht, New York, 2002.

[6] B. De Baets, Analytical solution methods for fuzzy relational equations, in: D. Dubois, H. Prade (eds.), The Handbook of Fuzzy Set Series Vol. 1, Academic Kluwer Publ., Boston, 2000, pp. 291-340.

[7] A. Di Nola, S. Sessa, W. Pedrycz, E. Sanchez, Fuzzy Relation Equations and Their Applications to Knowledge Engineering, Kluwer, Boston, 1989.

[8] D. Dubois, H. Prade, What are fuzzy rules and how to use them, Fuzzy Sets and Systems 84 (1996) 169-185.

[9] L. Godo, P. Hájek, A note on fuzzy inference as deduction, in: Fuzzy Sets, Logics and Reasoning about Knowledge (Applied Logic series), vol. 15, Kluwer Academic Publishers, 1999, pp. 237-241.

[10] S. Gottwald, Generalized solvability behaviour for systems of fuzzy equations, in: V. Novák, I. Perfilieva (eds.), Discovering the World with Fuzzy Logic, Studies in Fuzziness and Soft Computing, Springer-Verlag, Heidelberg, 2000, pp. 401-430. 
[11] S. Gottwald, On a generalization of fuzzy relation equations, in: Proc. of 11th IPMU Conference, Paris, France, 2006.

[12] S. Gottwald, An abstract approach toward the evaluation of fuzzy rule systems, in: Fuzzy Logic. A Spectrum of Theoretical \& Practical Issues (Studies in Fuzzines and Soft Computing), Springer-Verlag, 2007, pp. 281-297.

[13] P. Hájek, Metamathematics of Fuzzy Logic, vol. 4 of Trends in Logic, Kluwer Academic Publishers, Dordrecht, 1998.

[14] B. Jayaram, On the law of importation $(x \wedge y) \rightarrow z \equiv(x \rightarrow(y \rightarrow z))$ in fuzzy logic, IEEE Transactions on Fuzzy Systems 16 (2008) 130-144.

[15] F. Klawonn, Fuzzy points, fuzzy relations and fuzzy functions, in: V. Novák, I. Perfilieva (eds.), Discovering the World with Fuzzy Logic, Springer, Berlin, 2000, pp. 431-453.

[16] F. Klawonn, J. L. Castro, Similarity in fuzzy reasoning, Mathware \& Soft Computing 2 (1995) 197-228.

[17] E. P. Klement, R. Mesiar, E. Pap, Triangular Norms, vol. 8 of Trends in Logic, Kluwer Academic Publishers, Dordrecht, 2000.

[18] G. Klir, B. Yuan, Fuzzy Sets and Fuzzy Logic, Prentice Hall, New Jersey, 1995.

[19] L. Kóczy, K. Hirota, Interpolative reasoning with insufficient evidence in sparse fuzzy rule bases, Information Sciences 71 (1993) 169-201.

[20] C. K. Lim, C. S. Chan, A weighted inference engine based on intervalvalued fuzzy relational theory, Expert Systems with Applications 42 (7) (2015) $3410-3419$.

[21] E. H. Mamdani, S. Assilian, An experiment in linguistic synthesis with a fuzzy logic controller, Int. J. Man-Mach. Stud. 7 (1975) 1-13.

[22] S. Mandal, B. Jayaram, Bandler-Kohout subproduct with Yager's classes of fuzzy implications, IEEE Transactions on Fuzzy Systems 22 (3) (2014) 469-482. 
[23] B. Moser, M. Navara, Fuzzy controllers with conditionally firing rules, IEEE Trans. Fuzzy Systems 10 (3) (2002) 340-348.

[24] M. Navara, M. Petrík, How to use controller with conditionally firing rules, in: M. Štěpnička, V. Novák, U. Bodenhofer (eds.), New Dimensions in Fuzzy Logic and Related Technologies. Proc. 5th EUSFLAT Conference, vol. 2, Ostrava, 2007.

[25] L. Nosková, Systems of fuzzy relation equation with inf- $\rightarrow$ composition: solvability and solutions, J. of Electrical Engineering 12(s) (2005) 69-72.

[26] V. Novák, S. Lehmke, Logical structure of fuzzy IF-THEN rules, Fuzzy Sets and Systems 157 (15) (2006) 2003-2029.

[27] V. Novák, I. Perfilieva, J. Močkoř, Mathematical Principles of Fuzzy Logic, Kluwer Academic Publishers, Boston, 1999.

[28] W. Pedrycz, Applications of fuzzy relational equations for methods of reasoning in presence of fuzzy data, Fuzzy Sets and Systems 16 (1985) $163-175$.

[29] I. Perfilieva, S. Lehmke, Correct models of fuzzy IF-THEN rules are continuous, Fuzzy Sets and Systems 157 (2006) 3188-3197.

[30] E. H. Ruspini, A new approach to clustering, Inf. Control 15 (1969) $22-32$.

[31] E. Sanchez, Resolution of composite fuzzy relation equations, in: Information and Control, vol. 30, Academic press, 1976, pp. 38-48.

[32] I. Turksen, Z. Zhong, An approximate analogical reasoning approach based on similarity measures, IEEE Transactions on Systems, Man and Cybernetics 18 (6) (1988) 1049 -1056.

[33] M. Štěpnička, On the satisfaction of Moser-Navara axioms for fuzzy inference systems, in: Proc. 24th IEEE Int. Conf. on Fuzzy Systems (FUZZ-IEEE), Vancouver, Canada, 2016.

[34] M. Štěpnička, B. De Baets, Interpolativity of at-least and at-most models of monotone single-input single-output fuzzy rule bases, Information Sciences 234 (2013) 16-28. 
[35] M. Štěpnička, B. De Baets, L. Nosková, On additive and multiplicative fuzzy models, in: M. Šťpnička, V. Novák, U. Bodenhofer (eds.), New Dimensions in Fuzzy Logic and Related Technologies. Proc. 5th EUSFLAT Conference, vol. 2, Ostrava, 2007.

[36] M. Štěpnička, B. De Baets, L. Nosková, Arithmetic fuzzy models, IEEE Transactions on Fuzzy Systems 18 (2010) 1058-1069.

[37] M. Štěpnička, B. Jayaram, On the suitability of the Bandler-Kohout subproduct as an inference mechanism, IEEE Transactions on Fuzzy Systems 18 (2) (2010) 285-298.

[38] M. Štěpnička, B. Jayaram, Interpolativity of at-least and at-most models of monotone fuzzy rule bases with multiple antecedent variables, Fuzzy Sets Systems 297 (2016) 26-45.

[39] M. Štěpnička, S. Mandal, Conditionally firing implicative rules, in: Proc. 16th World Congress of the International Fuzzy Systems Association (IFSA) and 9th Conference of the European Society for Fuzzy-Logic and Technology (EUSFLAT), vol. 89 of Advances in Intelligent Systems Research, Atlantis press, Gijón, 2015, pp. 42-48.

[40] S. Yan, M. Mizumoto, W. Qiao, Reasoning conditions on Kóczy's interpolative reasoning method in sparse fuzzy rule bases, Fuzzy Sets and Systems 75 (1995) 63-71.

[41] L. A. Zadeh, Outline of a new approach to the analysis of complex systems and decision processes, IEEE Trans. Syst. Man Cybern. 3 (1) (1973) 28-44. 